\title{
Variabilidad Espacial del Carbono Orgánico e Inorgánico del Suelo en la Comarca Lagunera, México
}

\author{
Pedro Yescas-Coronado, Vicente de Paul Álvarez-Reyna, Miguel Ángel Segura-Castruita, \\ Mario García-Carrillo, Vicente Hernández-Hernández, Guillermo González-Gervantes
}

Pedro Yescas-Coronado

Vicente de Paul Álvarez-Reyna

Mario García-Carrillo

Vicente Hernández-Hernández

Universidad Autónoma Agraria, Antonio Narro, Unidad Laguna-Programa de Posgrado en Ciencias Agrarias, Periférico y Carretera Sta. Fe, s/n. C.P. 27054, Torreón, Coahuila, México.

\section{Miguel Ángel Segura-Castruita dmilys5@hotmail.com \\ Instituto Tecnológico de Torreón-DEPI. Ca- rretera Torreón-San Pedro, $\mathrm{km}$ 7.5. Ejido Anna, C.P. 27170 , Torreón, Coahuila, México.}

\section{Guillermo González-Gervantes}

Centro Nacional de Investigación Disciplinaria en Relación Agua Suelo Planta Atmósfera, (CENID-RASPA) Desarrollo Km. 6.5 margen derecha canal Sacramento, C.P. 35150, Gómez Palacio, Durango, México.

BOL. SOC. GEOL. MEX. 2018

VOL. 70 NO. 3

P. $591-610$

http://dx.doi.org/10.18268/BSGM2018v70n3a2

Manuscrito recibido: Mayo 20, 2017

Manuscrito corregido: Octubre 9, 2017

Manuscrito aceptado: Noviembre 15, 2017

\section{RESUMEN}

Las reservas de carbono orgánico, inorgánico y total son controladas parcialmente por la exposición a las condiciones ambientales, la cobertura vegetal y el uso del suelo. La geoestadística ha ayudado a comprender la distribución de los almacenes de carbono (orgánico, inorgánico y total) en el suelo. Sin embargo, existe escasa explicación de los factores que la determinan, específicamente en zonas áridas, y de su variabilidad espacial. Los objetivos de este trabajo fueron determinar las reservas de carbono orgánico e inorgánico en suelos agrícolas de la Comarca Lagunera de México, establecer su distribución y variabilidad espacial mediante el método geoestadístico kriging ordinario y validar los mapas temáticos con datos reales. Las reservas de carbono orgánico, inorgánico y total del suelo de muestras georreferenciadas se examinaron mediante un análisis de la semivarianza; los datos geoestadísticos se interpolaron, para obtener mapas temáticos de predicción de las mismas. Posteriormente, se validaron los mapas con datos de campo. Las reservas de carbono orgánico, inorgánico y total del suelo mostraron variabilidad espacial, cuya dependencia espacial del almacén de carbono orgánico del suelo fue débil (82.4\%), mientras que la del carbono inorgánico y total fue moderada (26.6 y $35.0 \%$, respectivamente). Los mapas temáticos de los almacenes de carbono orgánico e inorgánico difirieron, teniendo el mapa de carbono inorgánico un error de 2.01 $\mathrm{Mg} \mathrm{ha}^{-1}$ y el de carbono orgánico 4.69 Mg $\mathrm{ha}^{-1}$. El uso y manejo de los suelos en esta área influyeron en la distribución espacial de las reservas de carbono, mientras que la fisiografía, el material parental y el clima intervinieron en la distribución espacial del carbono inorgánico.

Palabras clave: materia orgánica, carbonatos, geoestadística, zonas áridas.

\section{ABSTRACT}

Organic, inorganic and total soil carbon stocks are partially controlled by exposure to environmental conditions, vegetation cover and land use. Geostatistics has helped to understand the distribution of carbon stocks (organic, inorganic and total) in the soils. However, there is little explanation of factors that determine it, specifically arid regions, and their spatial variability. The objectives of this work were to determine the organic and inorganic carbon reserves in agricultural soils of the Comarca Lagunera in Mexico, to establish their distribution and spatial variability using ordinary kriging and to validate the thematic maps whit real data. The correlation spatial of organic, inorganic and total soil carbon stocks of georeferenced samples were examined by an analysis of semivariance; the data were interpolated to obtain thematic maps of prediction of the different carbon stocks. Subsequently, the maps were validated with field data. The organic, inorganic and total soil carbon stocks showed spatial correlation, which was weak for the soil organic carbon storage (82.4\%), and moderate for the inorganic and total carbon (26.6 and $35.0 \%$, respectively). The thematic maps of organic and inorganic carbon stores differed, with the inorganic carbon map having an error of $2.01 \mathrm{Mg} \mathrm{ha}^{-1}$, which was lower than that of organic carbon $(4.69 \mathrm{Mg}$ $\left.h \mathrm{a}^{-1}\right)$. The use and management of soils in this area influenced the spatial distribution of carbon stocks, while physiography, parent material and climate intervened in the spatial distribution of inorganic carbon.

Keyreords: Organic matter, carbonates, geostatistics, arid region. 


\section{Introducción}

La variabilidad y distribución espacial de las reservas de carbono orgánico e inorgánico en el suelo (COS y CIS, respectivamente), como propiedades edáficas, están controladas parcialmente por las condiciones ambientales, cobertura vegetal y el uso del suelo (Guo et al., 2006). Estos factores influyen en la velocidad de formación y transformación del carbono orgánico $(\mathrm{CO})$ y en los cambios del carbono inorgánico (CI) del suelo, procesos que desempeñan un rol en el aumento o reducción de las concentraciones de dióxido de carbono en la atmósfera (West y Marland, 2002; Singh et al., 2007). Por ello el interés creciente en cartografiar las variaciones del COS y CIS para proveer al usuario del suelo de herramientas que permitan hacer un uso racional del suelo (Jaramillo, 2012; Orton et al., 2016). La escala para cartografiar COS puede ser a nivel parcela, cuenca, zona agroecológica, región, país o continente (IIASA/FAO, 2012). El análisis de datos de perfiles de suelos mediante sistemas de información geográficos (SIG), es una alternativa para conocer la distribución espacial y vertical del COS, mismo que puede utilizarse para CIS. No obstante, hay escasez de datos georeferenciados (FAO, 2002), necesarios para aplicar técnicas geoestadísticas para conocer la distribución espacial del COS a diferentes escalas y mejorar las estimaciones espaciales (Paz-Pellat et al., 2016). En México se han realizado estudios que muestran la distribución espacial del COS a nivel país (Segura-Castruita et al., 2005; Etchevers et al., 2006; Paz-Pellat et al., 2012; Cruz y Paz, 2013). Para estimar el potencial de captura de carbono en el suelo en diferentes escenarios, es necesario establecer una línea base de carbono para evaluar posteriormente cómo las concentraciones se modifican en función del cambio de uso del suelo o de las prácticas de manejo (Batjes, 1999). Sin embargo, no se tiene certidumbre acerca de los cambios del COS ni del CIS, en un lugar determinado en regiones áridas y semiáridas (Luo et al., 2011). En un análisis de la distribución espacial y temporal de COS se concluyó que, independientemente de la metodología utilizada y sus bases de datos, las estimaciones realizadas tienen el defecto de no poderse validar porque no se han empleado modelos predictivos (Paz-Pellat et al., 2016). La información disponible se refiere principalmente a regiones templadas y tropicales (Segura-Castruita et al., 2005), pero las regiones áridas y semiáridas han sido menos estudiadas. $\mathrm{Al}$ respecto, Montaño et al. (2016) mencionaron cómo la alta heterogeneidad espacio-temporal de estas regiones, las condiciones ambientales, la comunidad vegetal y el uso del suelo afectan los almacenes y flujos del carbono. Información relacionada con los almacenes de CIS y su cartografía es escasa (Segura-Castruita et al., 2005; Guo et al., 2006), al igual que el comportamiento de su variabilidad espacial. Determinarla resulta importante, ya que la aplicación de enmiendas orgánicas se ha convertido en una práctica común en áreas con agricultura intensiva en los distritos de riego de regiones áridas y semiáridas de México. Por ejemplo, los suelos de cultivo del Distrito de Riego 017 en la Comarca Lagunera reciben, año con año, aproximadamente entre 80 y $120 \mathrm{Mg} \mathrm{ha}^{-1}$ de enmiendas orgánicas (Fortis-Hernández et al., 2009), pero el efecto de estas aplicaciones en las reservas y el comportamiento espacial del COS y del CIS es desconocido. La distribución espacial de las reservas de COS y CIS en la Comarca Lagunera podría establecerse con datos georreferenciados de muestras de suelo y la aplicación de métodos geoestadísticos; con ello se podrían generar modelos predictivos, así como establecer relaciones con las características climáticas y el uso del suelo. Los objetivos de este trabajo fueron determinar el COS y CIS en suelos agrícolas de la Comarca Lagunera de México, establecer su distribución y variabilidad espacial mediante el método geoestadístico kriging ordinario $(\mathrm{KO})$ y verificar la precisión de los mapas temáticos. 


\section{Materiales y métodos}

\section{1. ÁREA DE ESTUDIO}

El estudio se realizó en el municipio de Matamoros (Figura 1), Coahuila, México (25 $23^{\prime}$ y $25^{\circ} 48^{\prime}$ latitud norte y $103^{\circ} 23^{\prime}$ y $103^{\circ} 03^{\prime}$ longitud oeste), que se encuentra en la parte plana de un valle, con altitudes que varían de 1100 a 1150 m en las partes bajas planas, hasta $1580 \mathrm{~m}$ en las cimas de los cerros. El clima del municipio es un BW hw" (e'), correspondiente a muy seco o desértico, semicálido con lluvias en verano e invierno fresco y oscilación extremosa. La precipitación total anual promedio es de $250 \mathrm{~mm}$ y una evaporación total anual promedio de $2500 \mathrm{~mm}$ (García, 2004), como se muestra en el climograma del área de estudio (Figura 2). La vegetación está dominada por matorrales xerófitos, aunque las praderas y el bosque espinoso (mezquite) son comunes. La alta concentración de sales en los suelos de esta región determina la existencia de una vegetación halófita dominante en el paisaje. Las plantas de matorrales del desierto, incluidos los rosetófilos y micrófilos, ocurren en un patrón irregular, compuesto principalmente de Larrea tridentata y halófitas. La geología del área está caracterizada por su origen (periodo Cuaternario y Cretácico), donde las rocas ígneas de la Sierra Madre Occidental son la principal fuente de sedimentos aluviales, que fueron

$103^{\circ} 23^{\prime} \mathrm{W}$

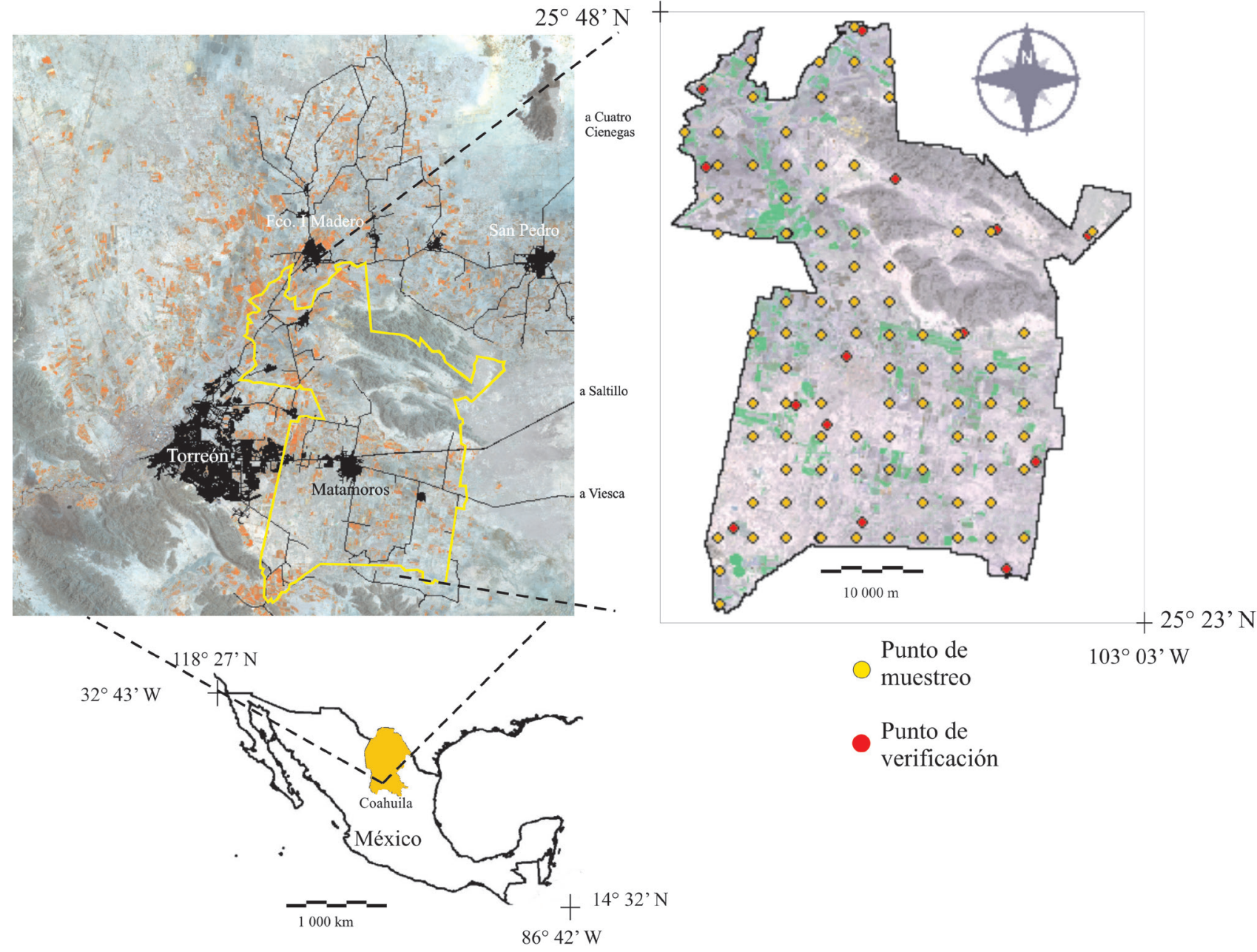


Estación meteorológica “El Cuije”, Lat. 25 41.8', Alt. 1120 msnm (record 29 años).

\begin{tabular}{|c|r|r|r|}
\hline Mes & $\mathrm{T}\left({ }^{\circ} \mathrm{C}\right)$ & $\mathrm{P}(\mathrm{mm})$ & $\mathrm{ETP}(\mathrm{mm})$ \\
\hline $\mathrm{E}$ & 15.7 & 8.4 & 98.8 \\
\hline $\mathrm{F}$ & 17.4 & 3.8 & 113.3 \\
\hline $\mathrm{M}$ & 20.1 & 0.9 & 167.7 \\
\hline $\mathrm{A}$ & 23.1 & 7.5 & 193.3 \\
\hline $\mathrm{M}$ & 26.1 & 23.9 & 231.1 \\
\hline $\mathrm{J}$ & 27.1 & 23.2 & 222.0 \\
\hline $\mathrm{J}$ & 27.2 & 28.4 & 225.6 \\
\hline $\mathrm{A}$ & 26.6 & 17.9 & 206.2 \\
\hline $\mathrm{S}$ & 25.7 & 26.6 & 175.1 \\
\hline $\mathrm{O}$ & 23.0 & 17.4 & 148.5 \\
\hline $\mathrm{N}$ & 19.0 & 9.1 & 112.4 \\
\hline $\mathrm{D}$ & 15.7 & 12.9 & 94.2 \\
\hline Anual & 22.2 & 180.0 & 1988.1 \\
\hline
\end{tabular}

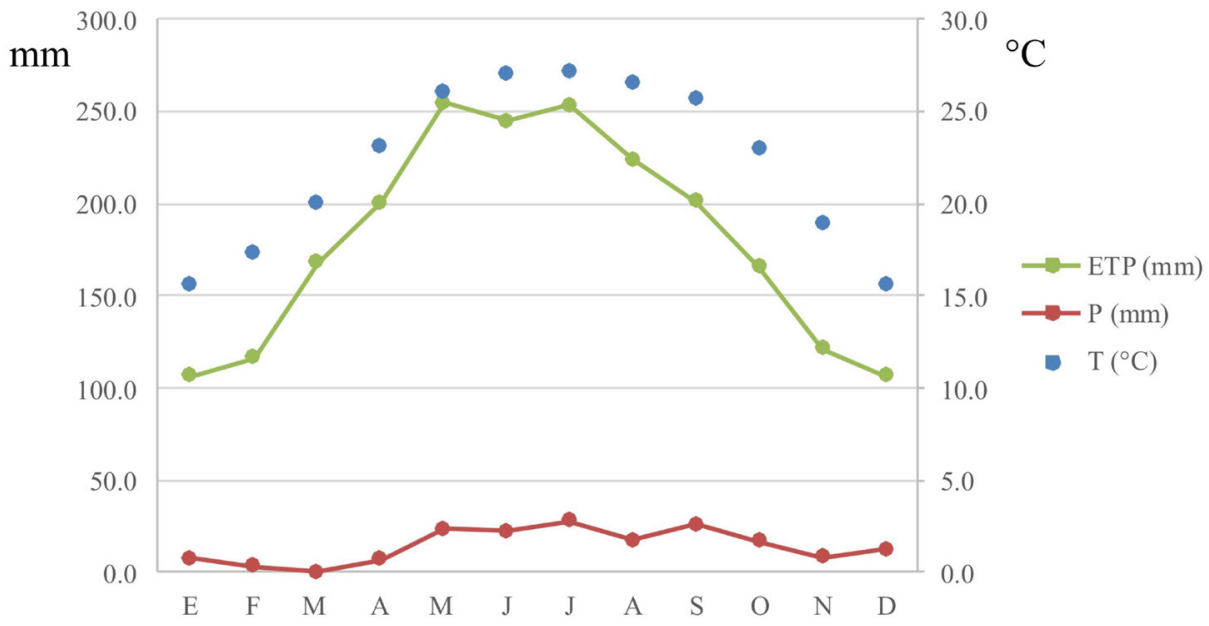

Figura 2 Climograma estación meteorológica El Cuije, municipio de Matamoros, Coahuila.

transportados por corrientes fluviales a través de los ríos Aguanaval y Nazas, que alimentaban el Paleolago Irritila (Figura 3a) en esta región (Czaja et al., 2014). La geomorfología de la cuenca (Figura 3b) se compone de depósitos tanto sedimentarios (calizas, dolomitas, yeso, arenas arcillosas y conglomerados) como de rocas ígneas (depósitos volcánicos y granito) (Mejía-González et al., 2014). Los sedimentos que se han identificado en dunas de la región están ligeramente redondeados e incluyen minerales como cuarzos, feldespatos, apatitas, micas, anfíboles (hornblenda), piroxenos y granos de magnetita, hematita y calcita; la forma ligeramente redondeada revela una corta distancia de transporte desde su procedencia (Czaja et al., 2014). Aparte, también se identifican sedimentos eólicos, así como afloramientos de calizas, yeso y conglomerados. Los suelos dominantes en el área del municipio que se han identificado son Feozems (60\%), Regosoles (20\%) Calcisoles (10\%) y Leptosoles, Fluvisoles y Vertisoles (INEGI, 2016a). El uso del suelo es principalmente agrícola y abarca el $68 \%$ de la superficie del municipio; también hay áreas urbanas $(7 \%$ ); la vegetación natural (área sin uso aparente) corresponde a matorrales y pastizales (INEGI, 2010).

\subsection{MÉTODOS}

El estudio se dividió en seis etapas: a) selección de los sitios de muestreo, b) muestreo de suelo, c) análisis de laboratorio, d) procesamiento de datos, e) validación de los mapas y f) análisis estadístico, que se describen a continuación.

\subsubsection{SELECGIÓN DE LOS SITIOS DE MUESTREO}

Una cuadrícula (2500 x 2500 m) con proyección UTM (Universal Transversa de Mercator) se sobrepuso en el polígono del área de estudio (Figura 1), con el fin de diseñar y realizar un muestreo sistemático. Cada vértice de la cuadrícula constituyó un sitio potencial de muestreo; se definieron 108 sitios. La ubicación de cada punto se registró para su posterior localización en campo. La elección final de los sitios de muestreo se condicionó al criterio de accesibilidad (carreteras pavimentadas, terracerías y brechas) a los lugares de interés, para lo cual se utilizó la carta topográfica de la región (INEGI, 2016b), quedando al final 92 sitios.

\subsubsection{MUESTREO DE SUELOS}

Una muestra única de dos kilogramos de suelo de la capa superficial (0 a $30 \mathrm{~cm})$ se recolectó en el punto seleccionado o lo más cercano a éste. Cada 

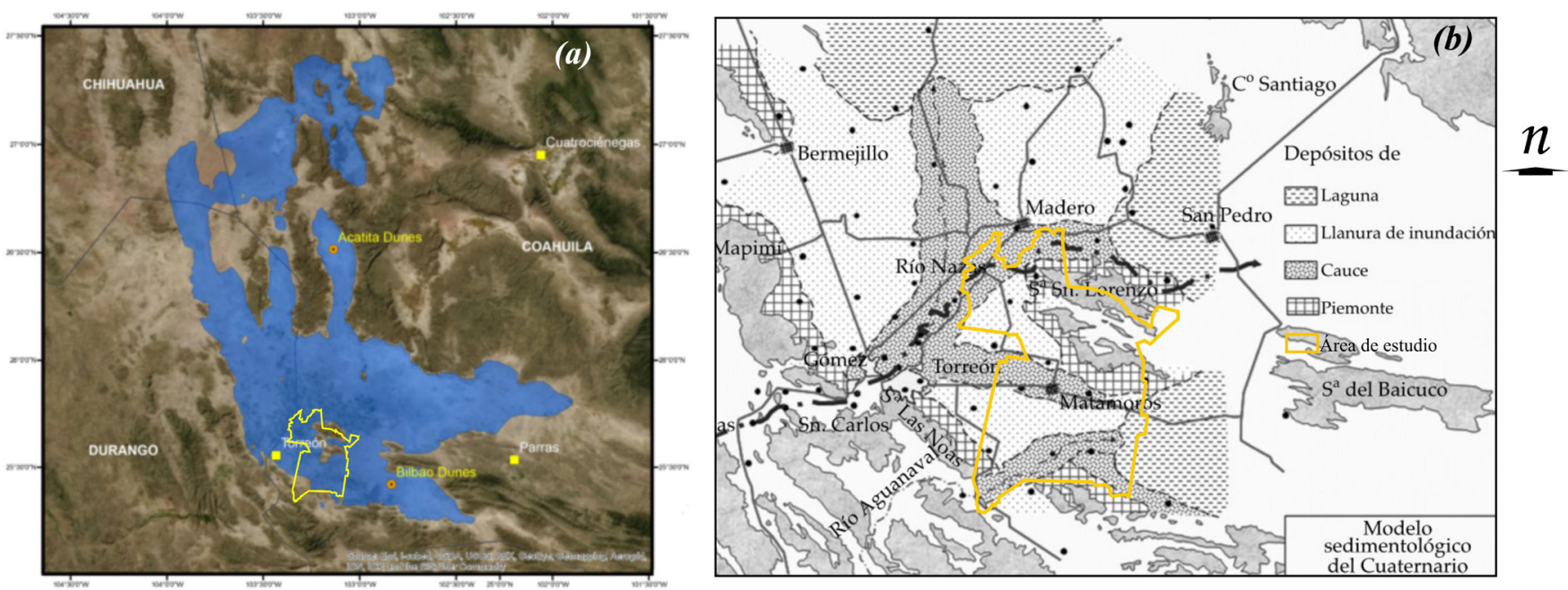

Figura 3 a) Perspectiva del área de estudio en el Paleolago Irritila (Adaptado del Czaja et al., 2014); b) Geomorfología del área de estudio en un modelo sedimentológico (Adaptado de Mejía-González et al., 2014).

punto se georreferenció en campo con un geoposicionador satelital (GPS) y se registró el uso de suelo y el tipo de manejo en cada sitio.

\subsubsection{ANÁLISIS DE LABORATORIO}

Las muestras se secaron a temperatura ambiente y a la sombra. Cuatro agregados de cada muestra de suelo se separaron para determinar densidad aparente (Da) por el método del terrón emparafinado; este método se utilizó porque, al extraer algunas muestras con el cilindro de núcleo, éstas se desmoronaban, lo que provocaría errores en los cálculos, pero presentaban agregados (terrones) de volumen indefinido, situación que se puede presentar en suelos suficientemente coherentes (SSS, 2014). El suelo restante se molió y tamizó, primero a través de una malla de $2 \mathrm{~mm}$, que sirvió para la determinación del carbonato de calcio equivalente (Horton y Newsom, 1953) y pH (en agua 1:2); otra parte de este suelo se tamizó en una malla de $0.5 \mathrm{~mm}$ de diámetro, antes de determinar el porcentaje de CO total (Walkley y Black, 1934); es importante aclarar que este método determina el porcentaje de materia orgánica $(\mathrm{MO})$ y se basa en la oxidación del carbono orgánico del suelo por medio de una disolución de dicromato de potasio $\left(\mathrm{K}_{2} \mathrm{Cr}_{2} \mathrm{O}_{4}\right)$ y el calor de reacción que se genera al mezclarla con ácido sulfúrico concentrado
$\left(\mathrm{H}_{2} \mathrm{SO}_{4}\right)$; después de un tiempo de espera la mezcla se diluye, se adiciona ácido fosfórico para evitar interferencias de $\mathrm{Fe}^{3+}$ y el dicromato de potasio residual es valorado con sulfato ferroso $\left(\mathrm{FeSO}_{4} 1 \mathrm{~N}\right.$ a $\mathrm{pH}$ 7). Este procedimiento detecta entre un 70 y $84 \%$ del carbón orgánico total, por lo cual es necesario introducir un factor de corrección, que puede variar entre suelo y suelo; en los suelos de México se utiliza el factor 1.2987 (1/0.77) (SEMARNAT, 2002). Posteriormente se estimó el CO total al dividir la MO entre el factor de Van Benmelen (1.724), que resulta del supuesto de que la $\mathrm{MO}$ contiene un $58 \%$ de CO. Los procedimientos se tomaron de la Norma Oficial Mexicana NOM021-REG NAT-2000 (SEMARNAT, 2002). Aparte, el porcentaje de carbono total (CT) del suelo se obtuvo por la suma aritmética del CO y CI. Los patrones y reactivos que se utilizaron fueron de la marca Merck ${ }^{\circledR}$. Los almacenes de COS, CIS y CTS se obtuvieron mediante la ecuación 1, propuesta por Ellert et al. (2006).

$$
\operatorname{CSD}_{i}=\frac{C S_{i} \times D a_{i} \times H_{i} \times\left(1-\eta_{i}\right)}{10}
$$

donde: $C S D_{i}$ representa los almacenes de COS, CIS o CTS en Mg ha ${ }^{-1}$ de $\mathrm{C}$ en la capa 0 - 30 cm de profundidad; $C S_{i}$ representa el contenido de CO, CI o CT $\left(\mathrm{g} \mathrm{kg}^{-1}\right)$ en la capa $i ; D a_{i}$ es la densi- 
dad aparente $\left(\mathrm{g} \mathrm{cm}^{-3}\right)$ de la capa $i$ y $H_{i}$ es su espesor $(\mathrm{cm})$. El término $\eta_{i}$ representa el porcentaje de las partículas con un tamaño $>2 \mathrm{~mm}$ en la capa, que se aplicó en aquellas muestras que presentaron este tamaño de partícula.

\subsubsection{PROGESAMIENTO DE DATOS}

Las coordenadas y los resultados de COS, CIS y CTS se registraron en una base de datos, que se utilizó para la realización de dos pasos. El primero consistió en un estudio estructural de las variables (COS, CIS y CTS) en el espacio con un análisis de la semivarianza, donde se analizó la similitud que existe entre observaciones a determinadas distancias estandarizadas llamadas retardos o lag (Jaramillo, 2012) con la ecuación (2).

$$
\gamma_{(h)}=\frac{1}{2 N_{(h)}} \sum_{i=1}^{N_{(h)}}\left[Z_{\left(x_{i}\right)}-Z_{\left(x_{i}+h\right)}\right]^{2}
$$

donde $\gamma_{(h)}$ es la semivarianza para COS, CIS o CTS de todas las muestras localizadas en el espacio por el intervalo de distancia $(h) ; \mathcal{N}_{(h)}$ es el número total de pares por un intervalo de distancia $(h)$; $Z_{x(x)}$ es el valor de la muestra en una localización $x i ; Z_{(x i+h)}$ es el valor de la muestra a la distancia del intervalo $h$ hasta $x$.

El número de lags en nuestro estudio fue de 15, con un tamaño para cada uno de $1700 \mathrm{~m}$, en una distancia activa de $25500 \mathrm{~m}$. A partir de este semivariograma experimental, las semivarianzas se ajustaron a un modelo esférico, ya que es el único que presenta sill o meseta verdadera, aspecto importante en la determinación del rango o alcance (Gallardo, 2006; Liu et al., 2006); también se ajustaron las semivarianzas a un modelo esférico porque se cuantificó la variabilidad espacial (semivariograma teórico) de las variables de estudio para su comparación. Los parámetros que se utilizaron en la interpolación y que ayudaron a explicar la variabilidad espacial del COS, CIS y CTS fueron el rango (alcance), nugget o efecto pepita $\left(C_{0}\right)$, sill o meseta $\left(C_{0}+C\right)$ y la varianza estructural $(C)$; también se obtuvieron el grado de dependencia espacial $\left[\mathrm{Co}_{0} /\left(\mathrm{Co}_{0}+\mathrm{C}\right)\right]$ y la proporción de la varianza explicada por el espacio o grado de variación espacial $\left[C /\left(C_{0}+C\right)\right]$ (Gallardo, 2006; Jaramillo, 2012). Esta información se utilizó para generar modelos de predicción y mapas temáticos de almacenes de carbono mediante interpolaciones con $\mathrm{KO}$, útil en variables ambientales y agrícolas (Elbasiouny et al., 2014). Los procedimientos se llevaron a cabo con el módulo Geoestatistical Analyst del software ArcMap $10.3^{\circ}$ (ESRI, 2014). Asimismo, en cada mapa de predicción $\left(\mathrm{COS}_{\mathrm{p}}\right.$, CIS $_{p}$ y CTS $_{\mathrm{p}}$ ) los almacenes de carbono se agruparon en cinco clases. También, en este módulo se realizó el segundo paso, que consistió en una validación cruzada de los valores observados (COS, CIS y CTS) y los obtenidos con los modelos de predicción o valores predichos $\left(\mathrm{COS}_{\mathrm{p}}\right.$, CIS y CTS $_{\mathrm{p}}$ ) para obtener los estadísticos: coeficiente de determinación $\left(R^{2}\right)$, media del error de la predicción $\left(M E_{p}\right)$, raíz cuadrada media del error de la predicción $\left(R C M E_{p}\right)$, media del error estandarizado de la predicción $\left(M E E_{p}\right)$, promedio del error estándar de la predicción $\left(P E E_{p}\right)$ y raíz cuadrada media del error estandarizado de la predicción $\left(R C M E E_{p}\right)$, con el fin de diagnosticar si los modelos fueron útiles en la elaboración de mapas predictivos (ESRI, 2014).

\subsubsection{VALIDACIÓN DE LOS MAPAS}

La validación de los mapas de predicción se verificó en 16 puntos que se seleccionaron en forma digital con un muestreo simple aleatorio estratificado en el área de estudio (Brus y de Gruijter, 2011; Segura-Castruita et al., 2014), procedimiento que se realizó con el módulo "Sample" (Random) de Idrisi-Selva (Eastman, 2013); se registraron las coordenadas y el valor del píxel de cada sitio en los mapas temáticos correspondientes, que representaban el carbono predicho $\left(\mathrm{COS}_{\mathrm{p}}\right.$ y $\left.\mathrm{CIS}_{\mathrm{p}}\right)$. Posteriormente, se acudió a campo y se colectó una muestra de suelo de la capa superficial de cada sitio seleccionado, misma que se llevó a laboratorio y se le determinó el porcentaje de $\mathrm{CO}$, CI y Da para calcular el almacén o la reserva de COS y CIS a una profundidad de $30 \mathrm{~cm}$. Estos resultados se compararon con los valores predichos. Finalmente, se elaboró una matriz de error. 


\subsubsection{ANÁLISIS ESTADÍSTICO}

Se calcularon los estadísticos descriptivos (media, máxima, mínima, desviación estándar y coeficiente de variación) de la $\mathrm{Da}$ y los porcentajes de $\mathrm{CO}$ y CI, así como de los almacenes de COS y CIS, además, para estas últimas se calcularon la asimetría y la curtosis. El desempeño de los modelos en comparación con datos de campo se estimó mediante índices estadísticos como la raíz cuadrada media del error (RCME) y el error medio del sesgo (EMS) con las siguientes ecuaciones (Douglas et al., 2009):

$$
R C M E=\left((1 / n) \sum_{1}^{n}(y-\hat{y})^{2}\right)^{0.5}
$$

donde $R C M E$ es la raíz cuadrada de la media del error en $\mathrm{Mg} \mathrm{ha}^{-1}$, y es el almacén de carbono predicho que se estimó con los modelos predictivos para cada mapa temático (en su caso $\mathrm{COS}_{\mathrm{p}}$, $\mathrm{CIS}_{\mathrm{p}}$ o CTS $_{\text {p }}$ que se evaluaron en este estudio, $\hat{y}$ es el almacén de carbono medido (COS, CIS y CTS), correspondiente a las muestras de suelo en los puntos de verificación.

$$
E M S=\left((1 / n) \sum_{1}^{n}(y-\hat{y})\right)
$$

donde $E M S$ es el error medio del sesgo en $\mathrm{Mg} \mathrm{ha}^{-1}$, y el resto de los símbolos indican lo mismo que en la ecuación (3). El software que se utilizó para esta etapa fue el Minitab 17 (Minitab Inc., 2013).

\section{Resultados}

El número de sitios que se logró muestrear fue 92, ya que algunos de los originalmente planteados se encontraban en lugares inaccesibles o no correspondían a suelo (áreas pavimentadas o estanques). Los suelos muestreados tuvieron una Da que varió de 1.13 a $1.57 \mathrm{~g} \mathrm{~cm}^{-3}$ (Tabla 1), lo cual reflejó las diferencias físicas entre los suelos, como se pudo constatar de manera visual con el tamaño de partículas, ya que en la cercanía de los ríos (abanicos aluviales) se encontraron partículas gruesas (arenas medias y finas), mientras que en el resto del área (llanuras de inundación) las partículas eran más finas (limos y arcillas). Los valores de $\mathrm{pH}$ variaron de 7.15 a 9.45, suelos entre neutros y alcalinos, respectivamente. Los porcentajes de carbono de los suelos variaron en función de su forma química (Tabla 1), de tal manera que el CO promedio $(1.15 \%)$ fue menor que el CI promedio (4.72\%), comportamiento que se repitió en todos los suelos del área de estudio. Asimismo, las variables en estudio se ajustan a una distribución normal, ya que la asimetría y curtosis se encuentran dentro del intervalo que va de - 3 a 3 (donde se supone que se encuentra el $99.5 \%$ de los datos de las variables). Por otra parte, los puntos muestreados tuvieron diferentes usos del suelo (Tabla 2): el $72 \%$ con uso agrícola de los cuales se encontraban en descanso (hasta por 10 años) 23 sitios, sin uso aparente (26 $\%$ ) y urbano $(2 \%)$. Los suelos agrícolas sostenían al momento del muestreo cultivos de alfalfa (10 sitios), algodón (6 sitios), frijol (un sitio), maíz y sorgo forrajero (21 sitios) y hortalizas (6 sitios); algunos con uso intensivo con dos cultivos anuales (monocultivos o intercalaciones), otros con un cultivo anual (ciclo primavera-verano) y semiperennes o perennes. Toda el área agrícola es de riego (con agua de presa y pozo profundo), puesto que todo el municipio tiene un clima árido; asimismo, el $59.1 \%$ de esta área recibe enmiendas orgánicas de estiércol.

\subsection{VARIABILIDAD ESPAGIAL DEL GARBONO}

La variabilidad espacial entre COS, CIS y CTS no fue similar (Figura 4), aun cuando las semivarianzas $\left(\gamma_{(h)}\right)$ de cada atributo se ajustaron al modelo esférico con coeficientes de determinación mayores que 0.85 .

Los semivariogramas teóricos revelaron que las varianzas no explicadas por el modelo [nugget $(C o)]$ fueron menores que las sill $\left(C_{0}+C\right)$ correspondientes (Figura 4). No obstante, la varianza estructural (C) de la semivarianza de CIS y CTS fue mayor que el nugget respectivo (Tabla 3); en cambio, COS tuvo una $C$ de 0.72 , menor que su nugget (3.38). El rango o alcance de la semivarianza de COS, CIS y CTS fue grande (> $16000 \mathrm{~m}$ ), ocupando 63.49, 
Tabla 1. Estadísticas descriptivas de los contenidos y almacenes de carbono, densidad aparente y $\mathrm{pH}$.

\begin{tabular}{|l|c|c|c|c|c|c|c|c|c|}
\hline \multicolumn{1}{|c|}{ Variable } & $\boldsymbol{N}^{*}$ & $\bar{x}$ & $\sigma$ & $\bar{x} \pm \sigma$ & $\mathbf{C V}$ & Mínimo & Máximo & Asimetría & Curtosis \\
\hline $\mathrm{CO}(\%)$ & 92 & 1.15 & 0.57 & $(0.58-1.72)$ & 46.63 & 0.15 & 2.16 & -0.05 & -1.21 \\
\hline $\mathrm{CI}(\%)$ & 92 & 4.72 & 1.87 & $(2.65-6.59)$ & 39.69 & 2.21 & 11.26 & 0.85 & 0.32 \\
\hline $\mathrm{CT}(\%)$ & 92 & 5.87 & 2 & $(3.87-7.87)$ & 34.04 & 2.67 & 12.82 & 0.85 & 0.57 \\
\hline $\mathrm{pH}$ & 92 & 8.43 & 0.47 & $(7.96-8.90)$ & 5.66 & 7.15 & 9.45 & -0.57 & 0.41 \\
\hline $\mathrm{Da}\left(\mathrm{g} \mathrm{cm}^{-3}\right)$ & 92 & 1.25 & 0.09 & $(1.16-1.34)$ & 7.43 & 1.13 & 1.57 & 0.45 & 0.97 \\
\hline $\mathrm{COS}\left(\mathrm{Mg} \mathrm{ha}^{-1}\right)$ & 92 & 4.27 & 2.01 & $(2.26-6.28)$ & 47.66 & 0.58 & 7.98 & -0.07 & -1.1 \\
\hline $\mathrm{CIS}\left(\mathrm{Mg} \mathrm{ha}^{-1}\right)$ & 92 & 17.7 & 7.1 & $(10.60-24.80)$ & 40.07 & 8.39 & 42.21 & 0.74 & 0.05 \\
\hline $\mathrm{CTS}\left(\mathrm{Mg} \mathrm{ha}^{-1}\right)$ & 92 & 21.97 & 7.35 & $(14.62-29.32)$ & 33.48 & 10.79 & 48.06 & 0.69 & 0.36 \\
\hline
\end{tabular}

$N^{*}$ : número de muestras, $\bar{x}$ : promedio, $\sigma$ : desviación estándar, $\bar{x} \pm \sigma$ : promedio más menos desviación estándar, CV: coeficiente de variación.

85.76 y $70.78 \%$, respectivamente, de la distancia efectiva de estudio (25500 m). Éstas fueron longitudes mayores en todos los casos a las distancias de muestreo ( $2500 \times 2500 \mathrm{~m}$ ), lo que muestra que la dependencia espacial de las variables ocurre en parte del área de estudio y no en el total de la misma, aunque dicha dependencia es mayor en el caso de CIS (21870.31 m). Tal situación se constató con el grado de dependencia espacial [Co/ $\left.\left(C_{0}+C\right)\right]$ que varió en los tres casos (Tabla 3); el CIS y CTS tuvieron moderada dependencia espacial, pero en el COS fue débil. En cambio, la varianza explicada por el espacio o grado de variación espacial $\left[C /\left(C_{0}+C\right)\right]$ para CIS y CTS fue mayor que $50 \%$, superior que la obtenida para el COS $(17.56 \%)$, lo que indica que CIS es más predecible espacialmente y por lo tanto la incertidumbre al interpolar los datos es menor.

La validación cruzada mostró que el modelo para $\mathrm{COS}_{\mathrm{p}}$ tuvo un $R^{2}=0.7602$, menor que en $\operatorname{CIS}_{\mathrm{p}}\left(R^{2}\right.$ $=0.9460)$. No obstante, los modelos de predicción tuvieron errores bajos después de la validación (< $0.05)$, ya que sus $M E_{p}$ y la $M E E_{p}$ fueron cercanos a cero (Tabla 4), donde la $M E E_{p}$ de la variable $\mathrm{COS}_{\mathrm{p}}$ fue más baja (0.0003). Estos resultados indican que, en los tres casos, las predicciones no se ajustan a los valores medidos. Al evaluar la incertidumbre de las predicciones se tuvo que la $R C M E_{p}$ del COS (4.6597) fue mayor que el $P E E_{p}$ (1.9697), esto significa que se subestimó la variabilidad de las predicciones; este resultado se confirmó al verificar que la $R C M E E_{p}$ (1.0216) fue mayor que uno, situación inversa en CIS y CTS, que tuvieron una $R C M E_{p}$ menor que el $P E E_{p}$, lo que indica una sobreestimación en la variabilidad de las predicciones, como se observó en el $R C M E E_{p}$ que fue menor que uno.

\subsection{DISTRIBUGIÓN ESPAGIAL DE LOS ALMAGENES DE GARBONO}

El mapa temático de COS presentó el mayor almacenamiento $\left(>4.5 \mathrm{Mg} \mathrm{ha}^{-1}\right.$ ) en el norte (Figura $5 a)$, área donde se encuentran suelos que reciben enmiendas orgánicas (80 - $120 \mathrm{Mg} \mathrm{ha}^{-1}$ cada año), mientras que las reservas más bajas se localizaron principalmente al noreste $\left(<2.5 \mathrm{Mg} \mathrm{ha}^{-1}\right)$. En cambio, el carbono inorgánico se encontró en franjas irregulares (Figura 5b), donde las menores acumulaciones se hallaron al noroeste y parte del centro $\left(<10.5 \mathrm{Mg} \mathrm{ha}^{-1}\right)$, que son lugares con uso agrícola intensivo y con aplicaciones de enmiendas orgánicas; por el contrario, las mayores acumulaciones se encontraron en sitios sin uso aparente (suelos que los productores conocen como arenales), con vegetación de matorral desértico o con diez años de descanso (con presencia de matorrales), que se ubican en la parte sur (14.5 a $\left.27 \mathrm{Mg} \mathrm{ha}^{-1}\right)$ y en la porción sureste $\left(>27 \mathrm{Mg} \mathrm{ha}^{-1}\right)$, en las llanuras de inundación y en el abanico aluvial del río Aguanaval, respectivamente, a una altitud que variaba de 
Tabla 2. Sitios de muestreo con coordenada UTM y su uso del suelo y manejo.

\begin{tabular}{|c|c|c|c|c|c|c|c|c|c|c|c|}
\hline ID & $\mathbf{s}^{\dagger}$ & $\mathbf{X}$ & $\mathbf{Y}$ & Uso & Manejo & ID & $\mathbf{s}^{\dagger}$ & $\mathbf{X}$ & $\mathbf{Y}$ & Uso & Manejo \\
\hline 1 & 1 & 677631 & 2849930 & Agrícola & 1AwOm & 47 & 87 & 685069 & 2825029 & Agrícola & $2 \mathrm{Tph}$ \\
\hline 2 & 4 & 670063 & 2847386 & Agrícola & $2 \mathrm{Trm}$ & 48 & 88 & 689988 & 2825023 & Agrícola & 1Apa \\
\hline 3 & 5 & 675065 & 2847381 & Sin uso & - & 49 & 90 & 670116 & 2822509 & Sin uso & - \\
\hline 4 & 6 & 677612 & 2847395 & Agrícola & $1 \mathrm{ArOm}$ & 50 & 91 & 672634 & 2822510 & Sin uso & - \\
\hline 5 & 7 & 680138 & 2847402 & Agrícola & $2 \mathrm{TD}$ & 51 & 92 & 675112 & 2822503 & Sin uso & - \\
\hline 6 & 9 & 670127 & 2844854 & Agrícola & 1ApOm & 52 & 94 & 680150 & 2822481 & Agrícola & $1 \mathrm{ApOm}$ \\
\hline 7 & 10 & 675078 & 2844860 & Agrícola & $2 \operatorname{Trs}$ & 53 & 95 & 682543 & 2822504 & Agrícola & $2 \mathrm{TD}$ \\
\hline 8 & 12 & 680132 & 2844890 & Agrícola & $2 \mathrm{TD}$ & 54 & 96 & 685080 & 2822493 & Agrícola & $2 \mathrm{TD}$ \\
\hline 9 & 13 & 665174 & 2842326 & Agrícola & 1ApOm & 55 & 97 & 687570 & 2822490 & Agrícola & $2 \mathrm{TD}$ \\
\hline 10 & 14 & 667582 & 2482337 & Agrícola & $1 \mathrm{ApOs}$ & 56 & 98 & 689969 & 2822504 & Sin uso & - \\
\hline 11 & 16 & 672592 & 2842336 & Agrícola & $2 \operatorname{Trg}$ & 57 & 99 & 670135 & 2820008 & Sin uso & - \\
\hline 12 & 20 & 667596 & 2839877 & Agrícola & $1 \mathrm{TpOg}$ & 58 & 100 & 672649 & 2820027 & Agrícola & $1 \mathrm{ApOm}$ \\
\hline 13 & 21 & 670094 & 2839827 & Agrícola & $2 \mathrm{Trm}$ & 59 & 101 & 675133 & 2820003 & Sin uso & - \\
\hline 14 & 22 & 672621 & 2839821 & Agrícola & $1 \mathrm{Trs}$ & 60 & 102 & 677663 & 2820017 & Agrícola & $2 \mathrm{TD}$ \\
\hline 15 & 23 & 675112 & 2839838 & Agrícola & $2 \mathrm{TD}$ & 61 & 103 & 680163 & 2820010 & Sin uso & - \\
\hline 16 & 24 & 677588 & 2839848 & Agrícola & $2 \mathrm{TD}$ & 62 & 105 & 685065 & 2820018 & Agrícola & 1Apg \\
\hline 17 & 29 & 667611 & 2837427 & Agrícola & $2 \mathrm{TD}$ & 63 & 106 & 687572 & 2819996 & Agrícola & $2 \mathrm{TD}$ \\
\hline 18 & 31 & 672606 & 2837417 & Agrícola & $1 \mathrm{ApOa}$ & 64 & 107 & 689992 & 2820001 & Sin uso & - \\
\hline 19 & 32 & 675112 & 2837406 & Agrícola & 2 Twm & 65 & 110 & 672642 & 2817608 & Agrícola & $2 \mathrm{Trm}$ \\
\hline 20 & 42 & 667625 & 2834864 & Agrícola & $2 \mathrm{TD}$ & 66 & 111 & 675142 & 2817611 & Agrícola & $1 \mathrm{ApOa}$ \\
\hline 21 & 43 & 670073 & 2834903 & Agrícola & $2 \operatorname{Trf}$ & 67 & 112 & 677663 & 2817612 & Agrícola & $2 \mathrm{Tph}$ \\
\hline 22 & 44 & 672624 & 2834939 & Agrícola & 1ApOm & 68 & 113 & 680155 & 2817626 & Agrícola & $1 \mathrm{ApOa}$ \\
\hline 23 & 45 & 675081 & 2834950 & Agrícola & 1ApOm & 69 & 114 & 672583 & 2817615 & Agrícola & $2 \mathrm{TD}$ \\
\hline 24 & 46 & 677630 & 2834932 & Agrícola & $1 \mathrm{ApOa}$ & 70 & 115 & 685056 & 2817605 & Agrícola & $2 \mathrm{Tph}$ \\
\hline 25 & 49 & 685054 & 2834960 & Agrícola & $2 \mathrm{TD}$ & 71 & 116 & 687585 & 2817609 & Agrícola & 2 Tra \\
\hline 26 & 50 & 687554 & 2834958 & Sin uso & - & 72 & 117 & 689989 & 2817600 & Agrícola & 1Apg \\
\hline 27 & 53 & 695014 & 2834963 & Sin uso & - & 73 & 119 & 670151 & 2815132 & Sin uso & - \\
\hline 28 & 54 & 675090 & 2832407 & Agrícola & 2TD & 74 & 120 & 672649 & 2815102 & Sin uso & - \\
\hline 29 & 55 & 677636 & 2832420 & Agrícola & $1 \mathrm{ApOa}$ & 75 & 121 & 675151 & 2815104 & Sin uso & - \\
\hline 30 & 56 & 680141 & 2832430 & Agrícola & $2 \mathrm{TD}$ & 76 & 122 & 677656 & 2815113 & Agrícola & $1 \mathrm{Aph}$ \\
\hline 31 & 61 & 672617 & 2829909 & Agrícola & 1Arg & 77 & 123 & 681066 & 2815132 & Sin uso & - \\
\hline 32 & 62 & 675126 & 2829907 & Agrícola & $2 \mathrm{Trm}$ & 78 & 124 & 682558 & 2815124 & Sin uso & - \\
\hline 33 & 63 & 677627 & 2829921 & Agrícola & $2 \operatorname{Trs}$ & 79 & 125 & 685059 & 2815102 & Agrícola & $1 \mathrm{ApOa}$ \\
\hline 34 & 64 & 680147 & 2829912 & Agrícola & 2TD & 80 & 126 & 687567 & 2815104 & Sin uso & - \\
\hline 35 & 70 & 670123 & 2827524 & Agrícola & 1ApOm & 81 & 128 & 667651 & 2812636 & Sin uso & - \\
\hline 36 & 71 & 672640 & 2827520 & Agrícola & $2 \mathrm{TD}$ & 82 & 129 & 670149 & 2812652 & Sin uso & - \\
\hline 37 & 72 & 675128 & 2827510 & Agrícola & $2 \mathrm{Trm}$ & 83 & 130 & 672644 & 2812634 & Sin uso & - \\
\hline 38 & 73 & 677639 & 2827516 & Agrícola & 2 Trm & 84 & 131 & 675146 & 2812639 & Sin uso & - \\
\hline 39 & 74 & 680146 & 2827505 & Agrícola & $2 \mathrm{TD}$ & 85 & 132 & 677663 & 2812631 & Sin uso & - \\
\hline 40 & 75 & 682573 & 2827497 & Agrícola & $1 \mathrm{ApOa}$ & 86 & 133 & 680157 & 2812634 & Agrícola & $2 \mathrm{TD}$ \\
\hline 41 & 76 & 685047 & 2827496 & Sin uso & - & 87 & 134 & 682570 & 2812618 & Agrícola & 1Apa \\
\hline 42 & 78 & 689963 & 2827527 & Agrícola & $2 \mathrm{Trh}$ & 88 & 135 & 685068 & 2812599 & Agrícola & $2 \mathrm{TD}$ \\
\hline 43 & 81 & 672638 & 2825024 & Agrícola & $2 \operatorname{Trg}$ & 89 & 136 & 687572 & 2812621 & Agrícola & $2 \mathrm{TD}$ \\
\hline 44 & 84 & 680146 & 2825014 & Agrícola & $2 \mathrm{TD}$ & 90 & 137 & 689980 & 2812580 & Agrícola & $2 \mathrm{TD}$ \\
\hline 45 & 85 & 682551 & 2825013 & Sin uso & - & 91 & 138 & 667668 & 2810173 & Agrícola & 1ApOm \\
\hline 46 & 86 & 685069 & 2825022 & Sin uso & - & 92 & 142 & 667672 & 2807684 & Agrícola & $2 \mathrm{Tph}$ \\
\hline
\end{tabular}

$S^{\dagger}$ : sitio de muestreo; 1: pequeña propiedad, 2: ejidal; A: manejo altamente tecnificado; T: manejo tradicional; D: en descanso (más de 10 años); p: riego con agua de pozo; r: riego con agua rodada; w: riego con aguas residuales; O: enmienda orgánica; a: alfalfa; f: frijol; g: algodón; m: maíz; n: nogal; s: sorgo. 


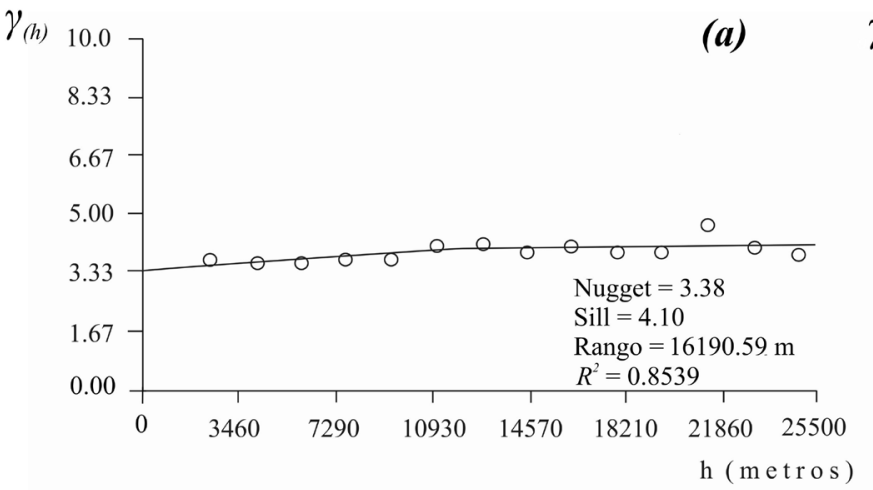

$\gamma_{(h)}$

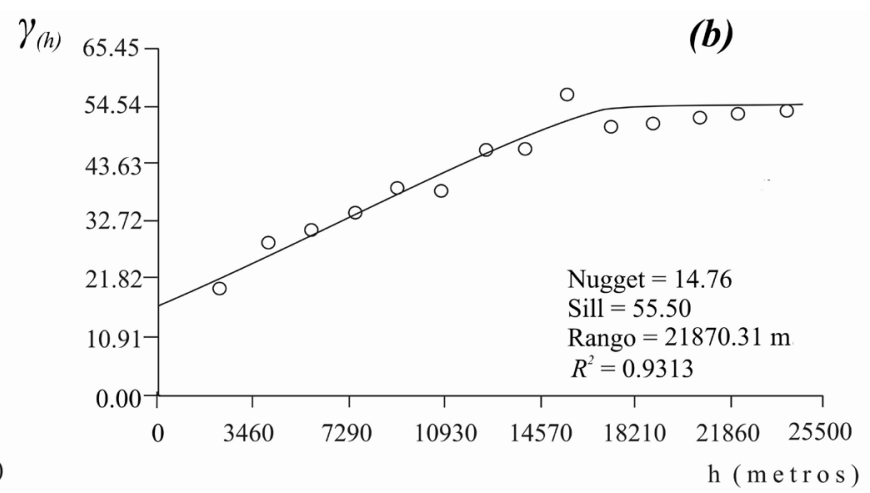

(c)

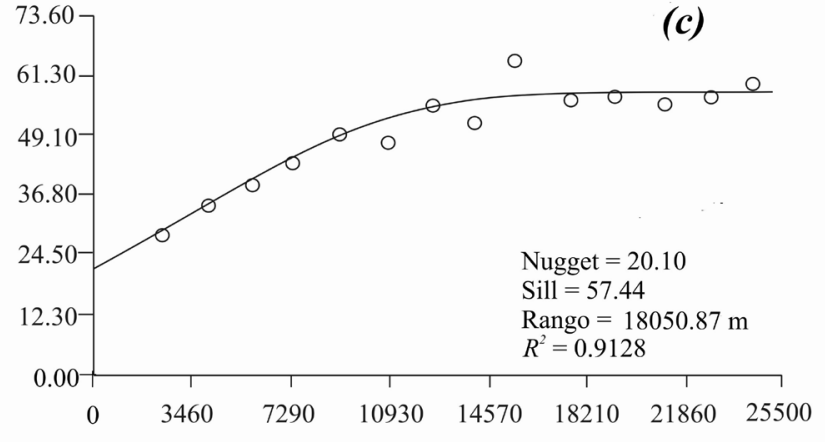

h (metros)

Figura 4 Semivariogramas de la semivarianza de los contenidos de carbono en los suelos de Matamoros, Coahuila; (a) carbono orgánico; (b) carbono inorgánico; (c) carbono total.

Tabla 3. Relaciones de la varianza estructural y nugget con el sill de las semivarianzas de los contenidos de carbono.

\begin{tabular}{|c|c|c|c|}
\hline Variable & & $\begin{array}{c}\boldsymbol{C} /(\boldsymbol{C o}+\boldsymbol{C}) \\
(\boldsymbol{\%})\end{array}$ & $\begin{array}{c}\boldsymbol{C} /(\boldsymbol{C o}+\boldsymbol{C}) \\
(\boldsymbol{\%})\end{array}$ \\
\hline COS & 0.72 & 17.56 & 82.44 \\
CIS & 40.74 & 73.4 & 26.6 \\
\hline CTS & 37.34 & 65 & 35 \\
\hline
\end{tabular}

${ }^{\dagger}$ Co: nugget, $C$ : varianza estructural, $C /(\mathrm{Co}+C)$ : grado de variación espacial, $\mathrm{Co} /(\mathrm{Co}+\mathrm{C})$ : grado de dependencia espacial.

1115 a 1150 m (Figura 6). En tanto que los contenidos de 10.5 a $12.5 \mathrm{Mg} \mathrm{ha}^{-1}$ se ubicaron en la parte media del municipio en sitios con suelos de textura media y fina, que se encontraron en los llanos de inundación del río Nazas a una altitud de 1110 a $1115 \mathrm{~m}$. Una distribución similar se tuvo para CTS (Figura 5c), donde los menores almacenamientos $\left(<14.5 \mathrm{Mg} \mathrm{ha}^{-1}\right)$ se ubicaron al norte, en el piedemonte de cerros de calizas y yeso, sin la influencia de los ríos mencionados, pues sus altitudes son mayores que 1130 metros. En el almacén de carbono total en el suelo (CTS), las reservas de CIS influyeron en un $79.5 \%$ promedio y con una correlación entre COS y CIS de tendencia negativa $(-0.016, p=0.88)$.

\subsection{VALIDAGIÓN DE LOS MAPAS}

Los mapas que muestran las reservas de COS y CIS (Figuras 5a y 5b, respectivamente) tuvieron precisiones parecidas en lo general (Tabla 5), aunque con ligeras diferencias, como se observa en los parámetros del error en la validación cruzada. Las RGME muestran que los valores predichos son mayores que las cantidades que se obtuvieron en campo para COS y CIS (0.6951 y $0.6461 \mathrm{Mg}$ ha-1, respectivamente); esto revela que los valores predichos podrían subestimar o sobreestimar los datos de campo. En cambio, los EMS de la verificación indican que los mapas de COS sobreestimaron los datos $\left(-0.1827 \mathrm{Mg} \mathrm{ha}^{-1}\right)$, mientras que el CIS subestimó los valores de campo $(0.0463 \mathrm{Mg}$ 
Tabla 4. Resultados de la validación cruzada.

\begin{tabular}{|c|c|c|c|c|c|c|c|}
\hline Variable & $\boldsymbol{N}^{\dagger}$ & $\boldsymbol{R}^{2}$ & $\boldsymbol{M E}_{\boldsymbol{p}}$ & $\boldsymbol{R C M \boldsymbol { E } _ { \boldsymbol { p } }}$ & $\boldsymbol{M E E _ { \boldsymbol { p } }}$ & $\boldsymbol{R C M E E _ { \boldsymbol { p } }}$ & $\boldsymbol{P E E _ { \boldsymbol { p } }}$ \\
\hline $\mathrm{COS}_{\mathrm{p}}$ & 92 & 0.7602 & 0.0079 & 4.6597 & 0.0003 & 1.0216 & 1.9697 \\
\hline $\mathrm{CIS}_{\mathrm{p}}$ & 92 & 0.946 & -0.0455 & 2.0158 & -0.0054 & 0.953 & 4.9752 \\
\hline $\mathrm{CTS}_{\mathrm{p}}$ & 92 & 0.9309 & -0.03 & 5.2717 & -0.0035 & 0.9502 & 5.6341 \\
\hline
\end{tabular}

$N^{\dagger}$ : número de muestras, $R^{2}$ : coeficiente de determinación, $M E_{p}$ : media del error de la predicción, $R C M E_{p}$ : raíz cuadrada media del error de la predicción, $M E E_{p}$ : media del error estandarizado de la predicción, $R C M E E_{p}$ : raíz cuadrada media del error estandarizado de la predicción, $P E E_{p}$ : promedio del error estandarizado de la predicción.
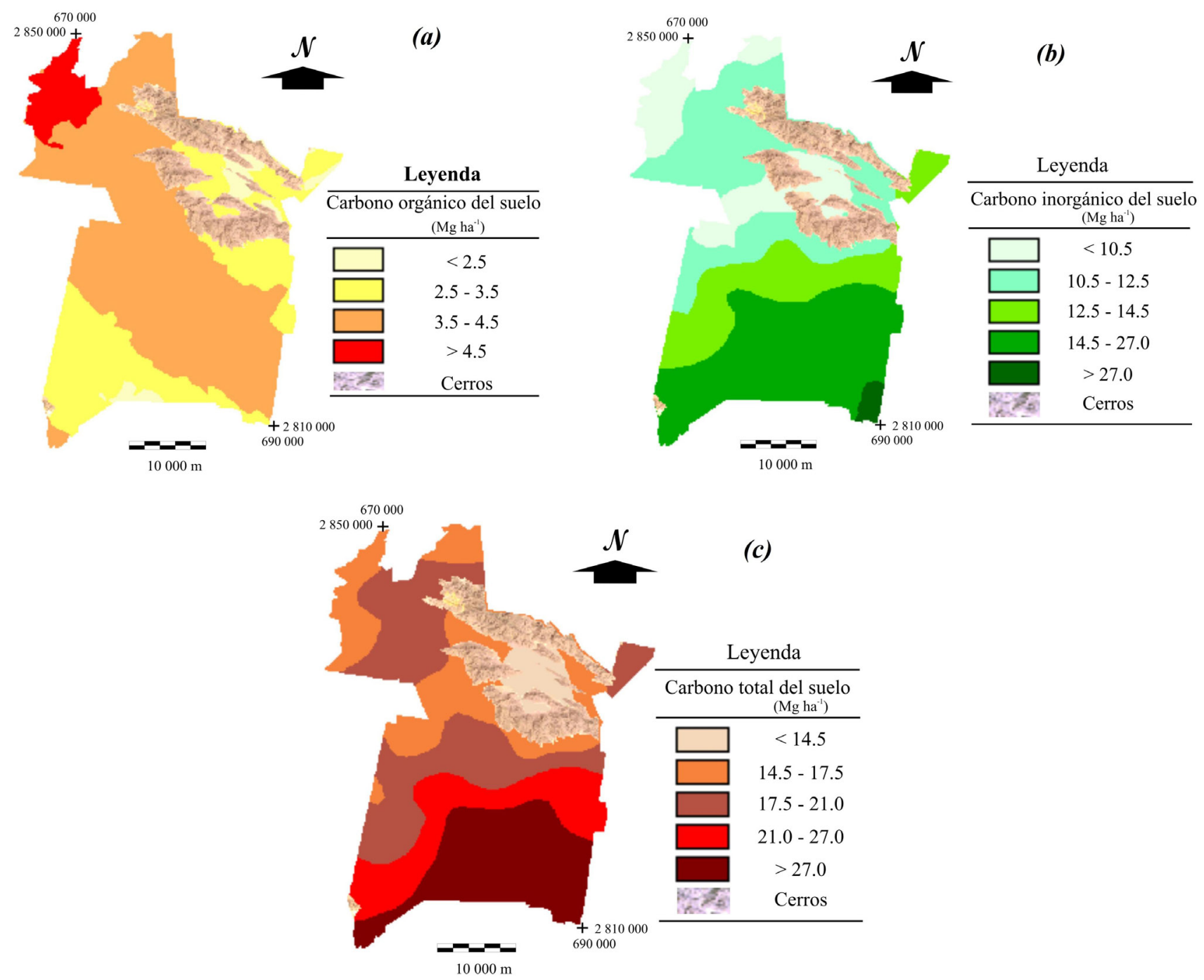

Figura 5 Mapas temáticos de contenido de carbono de Matamoros, Coahuila; (a) COS; (b) CIS; (c) CTS. 
(a)

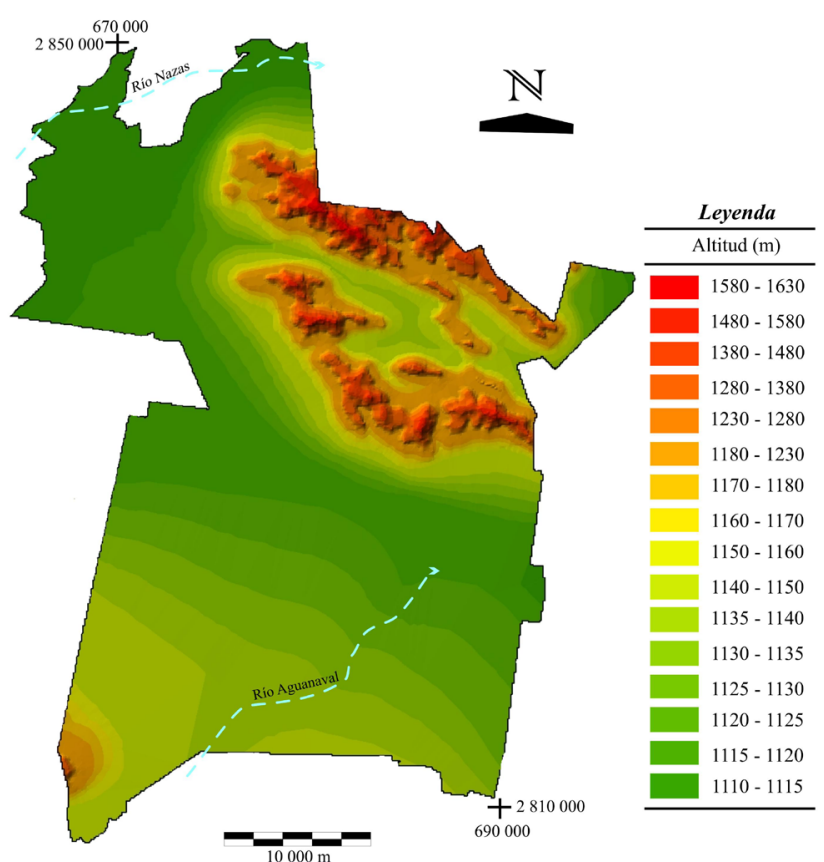

(b)
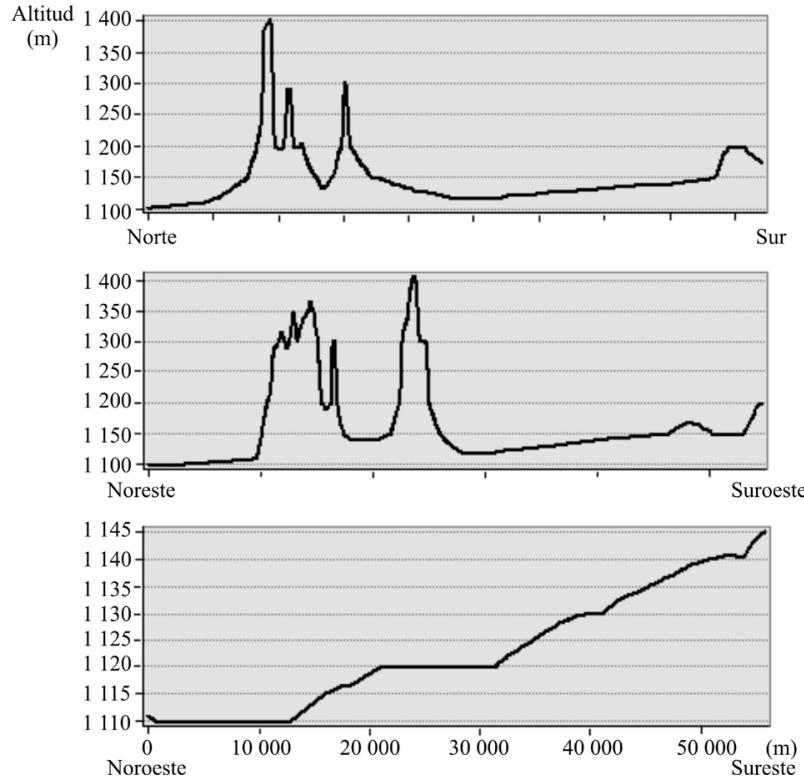

Figura 6 Planimetría de la fisiografía del municipio de Matamoros, Coahuila. (a) Curvas de nivel y la presencia de ríos; (b) perfiles topográficos en diferentes sentidos.

Tabla 5. Verificación de los mapas temáticos de COS y CIS predichos con los resultados de los contenidos de COS y CIS del segundo muestreo.

\begin{tabular}{|c|c|c|c|c|c|c|c|c|c|c|}
\hline \multirow{2}{*}{ No. } & \multicolumn{2}{|c|}{ UTM } & $\cos _{p}^{\dagger}$ & $\mathrm{CIS}_{\mathrm{p}}$ & $\mathrm{COS}_{\mathrm{m}}$ & $\mathrm{CIS}_{\mathrm{m}}$ & $\boldsymbol{R C M E _ { c o s }}$ & $E M S_{c o s}$ & $R C M E_{c i s}$ & $E M S_{c i s}$ \\
\hline & $\mathbf{X}$ & $\mathbf{Y}$ & \multicolumn{8}{|c|}{$\left(\mathrm{Mg} \mathrm{ha}^{-1}\right)$} \\
\hline 1 & 678077.91 & 2813794.18 & 3.72 & 26.66 & 4.1 & 25.75 & 0.1454 & 0.38 & 0.8197 & -0.91 \\
\hline 2 & 668791.06 & 2813483.92 & 3.37 & 20.35 & 3.35 & 19.86 & 0.0006 & -0.02 & 0.2353 & -0.49 \\
\hline 3 & 688706.19 & 2810484.71 & 4.04 & 26.54 & 3.54 & 27.01 & 0.2463 & -0.5 & 0.2245 & 0.47 \\
\hline 4 & 673228.11 & 2822584.97 & 4.21 & 16.89 & 3.83 & 16.98 & 0.1475 & -0.38 & 0.0079 & 0.09 \\
\hline 5 & 675601.42 & 2820930.28 & 4.29 & 19.79 & 4.01 & 20.11 & 0.0777 & -0.28 & 0.101 & 0.032 \\
\hline 6 & 666314.57 & 2845441.03 & 5.15 & 11.37 & 4.11 & 11.19 & 1.0841 & -1.04 & 0.032 & -0.18 \\
\hline 7 & 672608.99 & 2834892.08 & 4.68 & 13.43 & 4.33 & 14.67 & 0.1237 & -0.35 & 1.5438 & 1.24 \\
\hline 8 & 676942.85 & 2826101.29 & 4.33 & 15.35 & 3.84 & 15.33 & 0.2357 & -0.49 & 0.0003 & -0.02 \\
\hline 9 & 685549.37 & 2827957.48 & 3.51 & 12.9 & 4.25 & 13.84 & 0.5539 & 0.74 & 0.8749 & 0.94 \\
\hline 10 & 694691.05 & 2834788.66 & 2.9 & 15.92 & 2.51 & 15.53 & 0.1483 & -0.39 & 0.1507 & -0.39 \\
\hline 11 & 680564.61 & 2838924.48 & 4.52 & 13.18 & 3.68 & 13.48 & 0.7115 & 0.16 & 0.0926 & 0.3 \\
\hline 12 & 687880.69 & 2835202.34 & 3.23 & 12.6 & 3.69 & 13.54 & 0.2094 & 0.46 & 0.8777 & 0.94 \\
\hline 13 & 666314.57 & 2839959.71 & 5.3 & 11.8 & 4 & 11.26 & 0.2869 & -0.32 & 0.2868 & -0.54 \\
\hline 14 & 678077.91 & 2849681.29 & 4.93 & 11.53 & 3.45 & 11.15 & 2.1893 & -1.48 & 0.146 & -0.38 \\
\hline 15 & 675085.48 & 2812449.71 & 3.14 & 25.78 & 3.45 & 26.17 & 0.0955 & 0.31 & 0.1556 & 0.39 \\
\hline \multirow[t]{2}{*}{16} & 690666.78 & 2818241.29 & 4.61 & 23.22 & 4.88 & 22.16 & 0.0736 & 0.27 & 1.1284 & -1.69 \\
\hline & & & & & & & 0.6951 & -0.1827 & 0.6461 & 0.0463 \\
\hline
\end{tabular}

${ }^{\dagger} \mathrm{COS}_{\mathrm{p}}$ : carbono orgánico del suelo predicho, $\mathrm{CIS}_{\mathrm{p}}$ : carbono inorgánico del suelo predicho, $\mathrm{COS}_{\mathrm{m}}$ : carbono orgánico del suelo segundo muestreo, $\mathrm{CIS}_{\mathrm{m}}$ : carbono inorgánico del suelo segundo muestreo; RCME: raíz cuadrada media del error, EMS: error medio del sesgo. 
$\mathrm{ha}^{-1}$ ), aunque, específicamente, en los puntos de verificación tanto para COS como para CIS en ocasiones se subestimaron o sobreestimaron los almacenamientos de campo.

\section{Discusión}

El comportamiento de la semivarianza de COS, CIS y CTS y el ajuste del modelo esférico a los datos de los variogramas de este estudio, concuerdan con la distribución espacial de características edáficas (carbono orgánico, carbonatos, materia orgánica, $\mathrm{pH}, \mathrm{Ca}, \mathrm{P})$, reportadas en diferentes trabajos. En ellos se demuestra que los modelos esféricos tienen un mejor ajuste a los datos (Jaramillo, 2012; Bhunia et al., 2016; Sreenivas et al., 2016; Henríquez et al., 2013). Los nugget más bajos que los sill, revelaron una estructura espacial (PingguoYang et al., 2016), lo que refleja que los datos obtenidos en los muestreos son representativos de las variaciones espaciales (Axis-Arroyo et al., 2003). No obstante, la variabilidad espacial o estructural de CIS demuestra una mayor representatividad espacial que la presentada por COS, lo que indica que CIS sería espacialmente más predecible (Gallardo, 2006). Otro aspecto, los rangos de dependencia para cada variable, al resultar mayores que los distanciamientos de muestreo, revelan una relación espacial entre sus muestras. Ello sugiere un efecto de autocorrelación fuerte que puede deberse a algún factor de gran influencia (mayor a la distancia de muestreo) en el área de estudio, como lo indican Villatoro et al. (2008), que podría surgir de las características del suelo y de su manejo (Haws et al., 2004). Al considerar lo anterior y lo reportado acerca del material parental (Czaja et al., 2014), vegetación (INEGI, 2010), la fisiografía (Mejía-González et al., 2014) y el clima del área de estudio (Figura 2), el comportamiento de la variabilidad espacial de CIS podría deberse a estos factores formadores del suelo; en cambio, en COS, de acuerdo a la Tabla 2, la variabilidad se debería, principalmente, al uso del suelo (agrícola y sin uso aparente) y al manejo del mismo (intensivo con y sin enmiendas orgánicas) que se identificaron en el área, ya que entre puntos de muestreo hubo diferencias. Por otra parte, el grado de dependencia espacial o correlación de los datos fue clasificado por Cambardella et al. (1994) en tres grupos: fuerte $(<25 \%)$, moderada $(25-75 \%)$ y débil $(>75$ $\%$ ). La moderada dependencia espacial de CIS sugiere su correlación con otras características del suelo o el ambiente, como lo señalan PingguoYang et al. (2016), mientras que la débil dependencia espacial del COS fue reportada por Urricariet et al. (2011) y Delgado-Carranza et al. (2017) en estudios de distribución de carbono en suelos. Al respecto, Chien et al. (1997) mencionaron que el uso y manejo del suelo, aparte del tipo de suelo y clima, son factores que debilitan la dependencia espacial, de tal manera que la intervención de los humanos en la naturaleza provoca una disminución de la dependencia espacial del COS (Sun et al., 2003). La influencia del humano en el área de estudio se constató al identificar en la mayoría de los sitios de muestreo (Tabla 2) un uso agrícola con manejos diferentes (agua de riego, aplicación de fertilizantes, con o sin aplicaciones de enmiendas orgánicas, con uno o dos ciclos de cultivo anual), incluso en descanso, y por otra parte sitios sin uso aparente, con lo que se verificó la correspondencia entre los almacenes de COS y el manejo. Lo anterior permite deducir que el humano, como factor formador del suelo (Dudal, 2005), debilita o disminuye la dependencia espacial o la correlación de los datos de carbono, principalmente en regiones donde se encuentren tanto áreas naturales sin uso aparente como con actividades agrícolas, siendo más evidente en el COS que en el CIS, como se encontró en nuestro estudio.

En relación a la varianza explicada por el espacio o grado de variación espacial, Gallardo (2006) indicó que valores altos de esta varianza revelan que las variables en estudio pueden ser predecibles espacialmente y el grado de incertidumbre al momento de interpolar los datos es menor. De acuerdo con lo anterior, el CIS y el CTS serían espacialmente predecibles con un grado de incertidumbre bajo, pero no tanto en COS (Tabla 3); 
esto podría explicar las diferencias en los coeficientes de determinación de los modelos de predicción obtenidos en las validaciones cruzadas. Trabajos anteriores indicaron que la principal fuente de incertidumbre es la variabilidad en las reservas de COS, debida a errores en las determinaciones en laboratorio (Minasny et al., 2013), así como la variabilidad de los suelos y el contenido de fragmentos de roca (Goidts et al., 2009). En nuestro caso, como se mencionó anteriormente, la actividad humana a través del manejo del suelo, diferente en cada sitio, es otra fuente de incertidumbre, ya que influye principalmente en la variabilidad espacial del COS, mientras que en el CIS depende de los factores formadores del suelo (material parental, clima, vegetación y relieve) y sus relaciones, entendiendo éstas como los procesos formadores del suelo o procesos pedogenéticos como la carbonatación. Éste es la acumulación pedogenética de carbonatos en el suelo, que dependen del clima y el estado de desarrollo de los suelos (Raheb et al., 2017). En suelos de regiones áridas acumula carbonatos pedogenéticos de sedimentos no calcáreos, donde se combinan iones alcalinotérreos $\left(\mathrm{Ca}^{2+} \mathrm{y} \mathrm{Mg}^{2+}\right.$, principalmente), que provinieron de la disolución de minerales primarios, con agua de lluvia y $\mathrm{CO}_{2}$ de la respiración de las plantas (Drees et al., 2001) y se acumulan en las capas superficiales y subsuperficiales del suelo por la alta evaporación que existe en esas regiones (Cox, 2012), mientras que, a su vez, el relieve o fisiografia puede influir en su distribución. Este proceso puede acelerarse en suelos irrigados y cultivados (Sanderman, 2012) en esos climas. Por otra parte, los errores y la incertidumbre en el CTS se debieron a la manera como se obtuvieron sus valores, ya que fueron resultado de una suma aritmética de COS y CIS, lo que provocó que CTS acumulara los errores en las estimaciones de estos últimos.

\subsection{DISTRIBUGIÓN DEL GARBONO DEL SUELO}

Los mayores almacenes de COS, así como las menores de CIS, en algunos sectores del área agrícola se relacionaron con los sitios de prácticas de manejo con aplicación de enmiendas orgánicas de estiércol (Tabla 2), prácticas que de acuerdo con Fortis-Hernández et al. (2009), aplican hasta 120 $\mathrm{Mg} \mathrm{ha} \mathrm{p}^{-1}$ de estiércol. No obstante, los contenidos de COS fueron bajos en comparación con otras regiones áridas del mundo (Díaz-Hernández et al., 2003; Elbasiouny et al., 2014). Lentz y Lehrsch (2014) concluyeron que la adición de estiércol aumenta el almacenamiento de carbono en el corto plazo. No obstante, su manejo tradicional provoca la baja acumulación de carbono orgánico a largo plazo (Flores-Sánchez et al., 2015), ya que la degradación física causada por la labranza rompe los agregados y promueve la pérdida de COS (Pagliai et al., 2004), o como lo sugieren Raheb et al. (2017), el COS agregado decae rápidamente por su descomposición, promovida por la actividad de los microorganismos, y parte se convierte a CIS por reacciones químicas subsecuentes. Esta situación no ocurre en las áreas sin uso aparente, donde la vegetación de tipo matorral y las condiciones de aridez de la zona (INEGI, 2016a; Montaño et al., 2016) intervienen en la baja acumulación de COS. En contraste, la distribución del CIS está influenciada por las condiciones climáticas, que aunque son una constante en climas áridos como el del presente estudio, determinan la acumulación de carbonatos. A su vez, el material parental constituido de sedimentos aluviales (minerales de origen ígneo y sedimentario), a través de su alteración, contribuye con elementos alcalinotérreos que, por reacciones químicas (carbonatación), pueden formar carbonatos. Asimismo, la fisiografia, representada por la ubicación de los abanicos aluviales y las llanuras de inundación de los ríos Nazas y Aguanavala (Figura 3b) a diferentes altitudes (Figura 6), fue uno de los factores que más influyó en la distribución de los almacenes de CIS, ya que, en función del caudal de los ríos, el depósito de las partículas que transportaban fue en estas geoformas, como lo reveló la presencia de arenas medias y finas en el abanico aluvial del río Aguanaval. Un trabajo previo (Czaja et al., 2014) y la Figura 3a se tomaron como base para asumir que las corrientes fluviales del Nazas y el Aguanaval desembocaban en el Paleolago Irritila; de esta idea se sigue que la 
acumulación de carbonatos pudo deberse a su origen aluvial, lacustre o pedogenético, como se ha reportado en otras cuencas endorreicas de México y del mundo (Gutiérrez et al., 1998; Raheb et al., 2017), aspectos que en nuestro estudio no se determinaron. No hay que olvidar tampoco el factor humano, cuyas actividades agrícolas intensivas, por ejemplo, la adición de fertilizantes sintéticos, enmiendas orgánicas e incluso la calidad del agua de riego [como se presentó en el área de estudio (Tabla 2)] pueden inducir la modificación de los almacenes de CIS, lo que ya han indicado varios autores (Sartori et al., 2007; Sanderman, 2012; Lentz y Lehrsch, 2014), pero que no será abordado aquí pues excede los límites de la presente investigación.

Por otra parte, el aporte del CIS (79 \%) a las reservas de carbono total del suelo y la correlación con tendencia negativa con respecto a COS, fueron similares a las tendencias y aportes reportados en otras regiones áridas y semiáridas del mundo, donde el CIS contribuye con 84 y $74 \%$, respectivamente (Raheb et al., 2017). Lo anterior permite deducir que la capacidad de reserva de un suelo determinado podría presentar un límite de manera natural en regiones áridas, donde la disminución o aumento de un tipo de reserva (COS o CIS) se compensa con el otro a través del tiempo, siempre y cuando no intervenga el ser humano; de esta manera, para asegurar la permanencia o incremento de las reservas de carbono en el suelo, debería conocerse el origen y acumulación de COS y CIS, así como considerarse el tipo y calidad de las enmiendas orgánicas y el manejo o labranza que se utilicen en el lugar. Sin embargo, información relacionada con este aspecto es escasa, en regiones áridas.

\subsection{VALIDACIÓN DE LOS MAPAS TEMÁTICOS DE GARBONO}

Existen pocos estudios donde se valida la precisión de los mapas de carbono del suelo utilizando un método de muestreo aleatorio simple estratificado (Minasny et al., 2013). Brus et al. (2011) indicaron que el muestreo aleatorio simple estratificado es una buena opción para la verificación de la precisión de los mapas, ya que puede aumentar la precisión de las medidas estimadas de la calidad del mapa. En este sentido, el desempeño de un modelo en un periodo de tiempo dado se obtiene a través del RCME (Douglas et al., 2009) y su valor es siempre positivo (Yapo et al., 1998); a su vez, el EMS proporciona información del comportamiento a largo plazo de las correlaciones, lo que permite una comparación de la desviación real entre los valores pronosticados y medidos, término a término (Gunhan et al., 2005). De acuerdo con lo anterior, el mapa de CIS de nuestra investigación tuvo menor error y fue más preciso que el de COS, lo que fortalece los resultados que se encontraron en el análisis estructural de los almacenes de carbono.

\section{Conclusiones}

La distribución espacial de los almacenes de COS y CIS en la Comarca Lagunera se logró establecer con datos georreferenciados de muestras de suelo y la aplicación del método geoestadístico kriging ordinario, con lo que se generaron modelos de predicción de las reservas de carbono. Asimismo, las distribuciones espaciales de los almacenes de COS y CIS se relacionaron con los factores formadores del suelo para explicar su distribución. Los almacenes de carbono inorgánico de los suelos (CIS) de Matamoros, Coahuila, aportan una proporción de $79 \%$ al almacén de carbono total del suelo (CTS), cifra mayor a la que contribuye el carbono orgánico del suelo (COS) en este municipio. Igualmente, el análisis estructural de los almacenes de COS y CIS reveló que el CIS es más predecible espacialmente, ya que el grado de variación espacial $(73.40 \%$ ) fue mayor que en COS $(17.56 \%)$, por lo que el modelo de predicción de CIS tuvo un error menor que COS después de las validaciones cruzadas correspondientes $(2.0158 \mathrm{y}$ 4.6597, respectivamente), como se constató con la validación de estos modelos con datos reales. Los mapas de predicción mostraron la distribución es- 
pacial de las reservas de las fracciones de carbono. La distribución de COS está relacionada con el factor humano que interviene en áreas agrícolas a través del manejo, lo que influye directamente en su baja estructura espacial. En cambio, la distribución del CIS está relacionada principalmente con los factores formadores del suelo como la fisiografía, el material parental y el clima, ya que las reservas de CIS más grandes $\left(>27 \mathrm{Mg} \mathrm{ha}^{-1}\right)$ se encontraron en suelos de sedimentos gruesos sin uso aparente y con vegetación de matorral, que se ubicaron en el abanico aluvial del río Aguanaval, mientras que los almacenes con menores reservas se ubicaron en la parte norte, sobre las llanuras de inundación del río Nazas, en sitios con actividad agrícola intensiva. Finalmente, la relevancia de esta investigación radicó en el establecimiento de la distribución espacial del COS y del CIS en una zona árida de México y su relación con los factores formadores del suelo. No obstante, diferentes preguntas surgieron al concluir este trabajo, como ¿los suelos de regiones áridas tienen una máxima capacidad de reserva de carbono o es infinita?, ¿cuál es el origen de los carbonatos en esta región?, ¿aluvial, lacustre o pedogenético?, ¿cuáles son los mecanismos a utilizar para asegurar primero la permanencia de las fracciones de carbono en el suelo y después el incremento en aquellos suelos de uso agrícola?, entre algunas otras, lo que lleva a concluir que se requiere de más investigación en las regiones áridas para generar información que ayude a determinar el tipo de prácticas amigables para conservar el COS de los suelos, sin alterar el equilibrio entre los contenidos de COS y CIS e incluso que permita el incremento de las reservas de carbono.

\section{Agradecimientos}

Agradecemos al Tecnológico Nacional de México/Instituto Tecnológico de Torreón por el apoyo brindado para el uso de laboratorios y realización de análisis químicos, siendo este trabajo parte del proyecto 5654.15-P. Asimismo, se agradece a los revisores anónimos, así como al editor por sus comentarios y sugerencias que sin duda enriquecieron este documento.

\section{Referencias}

Axis-Arroyo, J., Mateu, J., Torruco, D., 2003, Diferencias entre modelos geoestadísticos aplicados en el análisis de la distribución espacio-temporal de especies biológicas, en Proceedings of the Gulf and Caribbean Fisheries 54, Islas Turcas y Caicos, Reino Unido, Gulf and Caribbean Fisheries Institute, 550-565.

Batjes, N.H., 1999, Management options for reducing $\mathrm{CO}_{2}$ concentrations in the atmosphere by increasing carbon sequestration in the soil: Wageningen, International Soil Reference and Information Centre, 200 p.

Bhunia, G.S., Shit, P.K., Maiti, R., 2016, Comparison of GIS-based interpolation methods for spatial distribution of soil organic carbon (SOC): Journal of the Saudi Society of Agricultural Science, 17(2), 114-126.

Brus, D.J., de Gruijter, J.J., 2011, Design-based generalized least squares estimation of status and trend of soil properties from monitoring data: Geoderma, 164, 172-180.

Cambardella, C.A., Moorman, T.B., Parking, T.B., Karlen, D.L., Novak, J.M., Turco R.F., Konopka, A.E., 1994, Field-scale variability of soil properties in central Iowa soils: Soil Science Society of America Journal, 58, 1501-1511.

Chien, Y.J., Lee, D.Y., Guo, H.Y., Houng, K.H., 1997, Geostatistical analysis of soil properties of mid-west TaiWan soils: Soil Science, 162, 291-298.

Cox, C.L., 2012, Evaluation of soil sustainability along the Rio Grande in West Texas: changes in salt loading and organic nutrients due to farming practices: El Paso, University of Texas at El Paso, 48 p. 
Cruz, C., Paz, F., 2013, Mapa de carbono orgánico de los suelos de la República Mexicana, en Paz, F., Bazan, M., Saynes, V. (eds.), Dinámica del Carbono en el Suelo 2012: Texcoco, Programa Mexicano del Carbono en colaboración con la Sociedad Mexicana de Ciencias del Suelo, 187-191.

Czaja, A., Palacios, F., M.R., Estrada, R., J.L., Romero, M., U., Alba, Á., J.A., 2014, Inland dunes fauna and flora from Paleolake Irritila in the Comarca Lagunera, Coahuila, northern Mexico: Boletín de la Sociedad Geológica Mexicana, 66, 541-551.

Delgado-Carranza, G., Bautista-Zuñiga, F., Calvo-Irabien, L.M., Aguilar-Duarte, Y.G., Martínez-Téllez, J.G., 2017, El carbono orgánico en Leptosols con distribución discontinua en la península de Yucatán: Ecosistemas y Recursos Agropecuarios, 4, 31-38.

Díaz-Hernández, J.L., Barahona, F.E., Linares, G., J., 2003, Organic and inorganic carbon in soils of semiarid regions: a case study from the Guadix-Baza basin (Southeast Spain): Geoderma, 114, 65-80.

Douglas, E.M., Jacobs, J.M., Sumner, D.M., Ray, R.L., 2009, A comparison of models for estimating potential evapotranspiration for Florida land cover types: Journal of Hydrology, 373, 366-376.

Drees, L.R., Wilding, L.P., Nordt, L.C., 2001, Reconstruction of soil inorganic and organic carbon sequestration across broad geoclimatic regions, en Lal, R. (ed.), Soil carbon sequestration and the greenhouse effect: Wisconsin, Soil Science Society American, 155-172.

Dudal, R., 2005, The sixth factor of soil formation: Eurasian Soil Science c/c of Pochvovedenie, 38, S60.

Eastman, J.R., 2013, IDRISI Guide to GIS and Image Processing in Idrisi Selva: Worcester, Clark University, 306 p.
Elbasiouny, H., Abowaly, M., Alkheirb, A.A., Gad, A.A., 2014, Spatial variation of soil carbon and nitrogen pools by using ordinary Kriging method in an area of north Nile Delta, Egypt: Catena, 113, 70-78.

Ellert, B.H., Janzen, H.H., Van den Bygaart, A.J., Bremer, E., 2006, Measuring change in soil organic carbon storage, en Carter, M.R., Gregorich, E.G. (eds.), Soil Sampling and Methods of Analysis: Boca Raton, CRC Press, 25-38.

Environmental Systems Research Institute (ESRI), 2014, ArcGis 10.3. Working with the ArcMap Spatial Analyst: California, ESRI Educational Services.

Etchevers, J.D., Masera, O., Balbontín, C., Gómez, D., Monterroso, A., Martínez, R., Acosta, M., Martínez, M., Ortiz, C., 2006, Soil carbon sequestration in Mexico and Central America (Biome A), en Lal, R., Cerri, C.C., Bernoux, M., Etchevers, J., Cerri, E. (eds.), Carbon Sequestration in Soils of Latin America: New York, Howarth Press, 119-146.

Flores-Sánchez, B., Segura-Castruita, M.A., Fortis-Hernández, M., Martínez-Corral, L., Aldaco-Nuncio, R.A., Orozco-Vidal, J.A., 2015, Enmiendas de estiércol solarizado en la estabilidad de agregados de un Aridisol cultivado de México: Revista Mexicana de Ciencias Agrícolas, 6, 1543-1555.

Food and Agriculture Organization (FAO), 2002, Captura de carbono en los suelos para un mejor manejo de la tierra: Roma, Organización de las Naciones Unidas para la Agricultura y la Alimentación, 61 p.

Fortis-Hernández, M., Leos, R., J.A., Orona, C., I., García, H., J.L., Salazar S., E., Preciado, R., P., Orozco, V., J.A., Segura, C., M.A., 2009, Uso de estiércol bovino en la Comarca Lagunera, en Orona, C., I., Salazar, S., E., Fortis, H., M., Trejo, E., H.I., Vázquez, V., G., (eds.), Libro de Agricultura Orgánica: Gómez Palacio, FAZ-UJED, 104-127. 
Gallardo, A., 2006, Geostadística: Ecosistemas, $15,48-58$.

García, E., 2004, Modificaciones al sistema de clasificación climática de Köppen: México, Instituto de Geografía-UNAM, 98 p.

Goidts, E., Van Wesemael, B., Crucifix, M., 2009, Magnitude and sources of uncertainties in soil organic carbon ( $\mathrm{SOC}$ ) stock assessments at various scales: European Journal of Soil Science, 60, 723-739.

Gunhan, T., Demir, V., Hancioglu, E., Hepbasli, A., 2005, Mathematical modeling of drying of bay leaves: Energy Conversion Management, 46, 1667-1679.

Guo, Y., Amundson, R., Gong, P., Yu, Q., 2006, Quantity and spatial variability of soil carbon in the conterminous United States: Soil Science of American Journal, 70, 590-600.

Gutiérrez, M.C., Stoops, G.G., Ortiz-Solorio, G.A., 1998, Carbonato de calcio en los suelos del Ex Lago de Texcoco: Terra, 16, 11-19.

Haws, N.W., Liu, B., Boast, C.W., Rao, P.S.C., Kladivko, E.J., Franzmeier, D.P., 2004, Spatial variability and measurement scale of infiltration rate on an agricultural landscape: Soil Science Society of America Journal, 68, 1818-1826.

Henríquez, C., Méndez, J.C., Masís, R., 2013, Interpolación de variables de fertilidad de suelo mediante el análisis kriging y su validación: Agronomía Costarricense, 37, 71-82.

Horton, J.H., Newsom, D.W., 1953, A rapid gas evolution method for calcium carbonate equivalent in liming materials: Soil Science Society of America Journal, 17, 414-415.

International Institute for Applied Systems Analysis/Food and Agriculture Organization (IIASA/FAO), 2012, Global Agro-Ecological Zones (GAEZ v3.0): Rome, International Institute for Applied Systems Analysis and FAO, 179 p.

Instituto Nacional de Estadística, Geografía e informática (INEGI), 2010, Carta Uso de Suelo y Vegetación Torreón G13-9, escala
1:250,000: México, Instituto Nacional de Estadística Geografía e Informática, 1 Mapa. Instituto Nacional de Estadística, Geografía e informática (INEGI), 2016a, Sistema de descarga de productos digitales, disponible en 'http://www.beta.inegi.org.mx/temas/ mapas/edafologia/>, consultado 31 enero 2016.

Instituto Nacional de Estadística, Geografía e informática (INEGI), 2016b, Sistema de descarga de productos digitales, disponible en 'http://www.beta.inegi.org.mx/temas/ mapas/topografico/ ${ }^{>}$, consultado 04 febrero 2016.

Jaramillo, D.F., 2012, Variabilidad espacial del suelo, bases para su estudio: Revista de la Facultad de Ciencias Universidad Nacional de Colombia, 1, 73-87.

Lentz, R.D., Lehrsch, G.A., 2014, Manure and fertilizer effects on carbon balance and organic and inorganic carbon losses for an irrigated cornfield: Soil Science Society of America Journal, 78, 987-1002.

Liu, D., Wang, Z., Zhang, B., Song, K., Li, X., Li, J., Li, F., Duan, H., 2006, Spatial distribution of soil organic carbon and analysis of related factors in croplands of the black soil region, Northeast China: Agriculture, Ecosystems and Environment, 113, 73-81.

Luo, Y., Durenkamp, M., De Nobili, M., Lin, Q., Brookes, P.C., 2011, Short term soil priming effects and the mineralisation of biochar following its incorporation to soils of different pH: Soil Biology and Biochemistry, 43, 2304-2314.

Mejía-González, M.Á., González-Hita, L., Briones-Gallardo, R., Cardona-Benavides, A., Soto-Navarro, P., 2014, Mecanismos que liberan arsénico al agua subterránea de la Comarca Lagunera, estados de Coahuila y Durango, México: Tecnología y Ciencias del Agua, 5, 71-82.

Minasny, B., McBratney, A.A., Malone, B.P., Wheeler, I., 2013, Digital mapping of soil carbon: Advances in Agronomy, 118, 4-51. 
Minitab Inc®, 2013, Minitab Inc.: State Collage, Minitab Inc.

Montaño, N.M., Ayala, F., Bullock, S.H., Briones, O., García-Oliva, F., García-Sánchez, R., Maya, Y., Perroni, Y., Siebe, C., Tapia-Torres, Y., Troyo, E., Yépez, E., 2016, Almacenes y flujos de carbono en ecosistemas áridos y semiáridos de México, Síntesis y perspectivas: Terra Latinoamericana, 34, 39-59.

Orton, T.G., Pringle, M.J., Bishop, T.F.A., 2016, A one-step approach for modelling and mapping soil properties based on profile data sampled over varying depth intervals: Geoderma, 262, 174-186.

Pagliai, M., Vignozzi, N., Pellegrini, S., 2004, Soil structure and the effect of management practices: Soil and Tillage Research, 79, 131-143.

Paz-Pellat, F., Hidalgo-Moreno, G., EtcheversBarra, J., 2012, Análisis y modelación de las energías de dispersión en la distribución del carbono orgánico en los suelos por fracciones físicas, en Paz, F., Cuevas, R. (eds.), Estado Actual del Conocimiento del Ciclo del Carbono y sus Interacciones en México: Síntesis a 2011: Texcoco, Programa Mexicano del Carbono en colaboración con la Universidad Autónoma del Estado de México y el Instituto Nacional de Ecología, 240-247.

Paz-Pellat, F., Covaleda, S., Hidalgo, C., Etchevers, J., Matus, F., 2016, Modelación simple y operativa de la distribución del carbono orgánico por fracciones físicas en los suelos: Terra latinoamericana, 34, 321-327.

PingguoYang, Byrne, J.M., Yang, M., 2016, Spatial variability of soil magnetic susceptibility, organic carbon and total nitrogen from farmland in northern China: Catena, 145, 92-98.

Raheb, A., Heidari, A., Mahmoodi, S., 2017, Organic and inorganic carbon storage in soils along an arid to dry sub-humid climosequence in northwest of Iran: Catena, $153,66-74$.
Sanderman, J., 2012, Can management induced changes in the carbonate system drive soil carbon sequestration? A review with particular focus on Australia: Agriculture, Ecosystems and Environment, 155, 70-77.

Sartori, F., Lal, R., Ebinger, M.H., Eaton, J.A., 2007, Changes in soil carbon and nutrient pools along a chronosequence of poplar plantations in Columbia Plateau, Oregon, USA: Agriculture, Ecosystems and Environment, 122, 325-339.

Secretaria del Medio Ambiente y Recursos Naturales (SEMARNAT), 2002, Norma Oficial Mexicana que establece las Especificaciones de Fertilidad, Salinidad y Clasificación de Suelos. Estudios, Muestreos y Análisis (NOM-021-RECNAT-2000): México, Diario Oficial de la Federación, 31 de diciembre, 2002, 85 p.

Segura-Castruita, M.A., Sánchez-Guzmán, P., Ortiz-Solorio, C.A., Gutiérrez-Castorena, M.C., 2005, Carbono orgánico de los suelos de México: Terra Latinoamericana, 23, 21-28.

Segura-Castruita, M.A., Huerta-García, A., Fortis-Hernández, M., Montemayor-Trejo, J.A., Martínez-Corral, L., Yescas-Coronado, P., 2014, Cartografía de la probabilidad de ocurrencia de Atriplex canescens en una región árida de México: Agrociencia, 48, 639-652.

Singh, S.K., Singh, A.K., Sharma, B.K., Tarafdar, J.C., 2007, Carbon stock and organic carbon dynamics in soils of Rajasthan, India: Journal of Arid Environments, 68, 408-421.

Soil Survey Staff (SSS), 2014, Kellogg soil survey laboratory methods manual. Soil survey investigations report No. 42, version 5.0: Nebraska, Department of Agriculture, Natural Resources Conservation Service, $1001 \mathrm{p}$.

Sreenivas, K., Dadhwal, V.K., Kumar, S., Harsha, G.S., Mitran, T., Sujatha, G., Janaki, G.R.S., Fyzee, M.A., Ravisankar, T., 2016, Digital mapping of soil organic and inorganic carbon status in India: Geoderma, 269, 160-173. 
Sun, B., Zhou, S., Zhao, Q., 2003, Evaluation of spatial and temporal changes of soil quality based on geostatistical analysis in the hill region of subtropical China: Geoderma, 115, 85-99.

Urricariet, S., Niborski, M., Kvolek, C.M., 2011, Atributos del suelo y paisaje asociados a la variabilidad de rendimientos de maíz en la pampa arenosa: Ciencia del Suelo, 29, 49-59.

Villatoro, M., Henríquez, G., Sancho, F., 2008, Comparación de los interpoladores IDW y Kriging en la variación espacial de pH, Ca, CICE y P del suelo: Agronomía Costarricense, 32, 95-105.
Walkley, A., Black, C.A., 1934, An examination of the Degtjareff method for determining soil organic matter and a proposed modification of chromic acid titration method: Soil Science, 37, 29-38

West, O., Marland, G., 2002, A synthesis of carbon sequestration, carbon emissions, and net carbon flux in agriculture: comparing tillage practices in the United States: Agriculture, Ecosystems and Environment, 91, 217-232.

Yapo, P.O., Gupta, H.V., Sorooshian, S., 1998, Multi-objective global optimization for hydrologic models: Journal of Hydrology, 204, 83-97. 